\title{
The Multi-Course Approach of Photodynamic Therapy to Treat Invasive Cervical Cancer IB2: A Case Report
}

\author{
Maxim Afanasiev ${ }^{\mathrm{a}} \quad$ Alexander Dushkin $^{\mathrm{b}}$ Tatyana Grishacheva $^{\mathrm{c}}$ \\ Stanislav Afanasiev ${ }^{d} \quad$ Yuri Nesvizhsky ${ }^{f} \quad$ Alexander V. Karaulov ${ }^{a}$ \\ Andrey Pylev ${ }^{\mathrm{e}}$
}

\begin{abstract}
aDepartment of Clinical Immunology and Allergology, I.M. Sechenov First Moscow State Medical University (Sechenov University), Moscow, Russian Federation; ${ }^{b}$ Department of Oncology, The Loginov Moscow Clinical Scientific Center is State Institution funded by Moscow Health Department, Moscow, Russian Federation; ' Laser Medicine Center, First Pavlov State Medical University of Saint Petersburg, Saint Petersburg, Russian Federation; ${ }^{\mathrm{d}}$ G.N. Gabrichevsky Moscow Research Institute for Epidemiology and Microbiology, Moscow, Russian Federation; 'Center for Innovative Medical Technologies "European Clinic", Moscow, Russian Federation; fDepartment of Microbiology, Virology and Immunology, I.M. Sechenov First Moscow State Medical University (Sechenov University), Moscow, Russian Federation
\end{abstract}

\section{Keywords}

Photodynamic therapy · Cervical cancer · Fertility-preserving surgery · Multi-course photodynamic therapy

\begin{abstract}
Cervical cancer is an important problem in women's health and a worldwide oncological disease. In 2018, the WHO registered 569,847 new cases in the world, and 3.4\% were in the Russian Federation. We describe here a case of invasive cervical cancer stage IB2 associated with human papilloma virus in a woman who was treated by multicourse photodynamic therapy (PDT). A 38-year-old woman presented with abdominal pain and genital tract spotting in October 2015. Colposcopy revealed a neoplasm in cauliflower form. PAP smear result was cancer in situ (Tis). The biopsy result from the cervical canal and neoplasm was invasive squamous cell carcinoma. The patient underwent full preoperative examination (blood test, biochemical blood test, coagulation test, urinalysis, X-ray of chest organs, ECG, ultrasound investigation of pelvic organs, and PAP smear). Magnetic resonance imaging investigation showed a heterogeneous tumor, uneven contours, and intensity accumulating contrast. The patient was not pregnant, and a fertility-preserving treatment method was used. Three PDT sessions allowed to avoid vaginal radical trachelectomy. Pregnancy occurred 3 years and 8 months after the first PDT session. The patient had testing after treatment 4 times (3rd, 12th, 24th, and 60th
\end{abstract}


months). She had a pregnancy without complications and had operative delivery by Cesarean section in April 2020. There was a 5-year remission period without episodes of relapse. The patient has an 8-month-old baby.

(C) 2021 The Author(s).

Published by S. Karger AG, Base

\section{Introduction}

Cervical cancer ranks 4th in the prevalence of all cancers in women. In 2018, the WHO registered 569,847 new cases in the world, and 3.4\% were in the Russian Federation. Cervix uteri cancer has the second rank in the world among female cancers in the fertile period [1]. IB2 is the last cervical cancer stage which is restricted to the cervix. The 5-year relative survival rate in the local stage is $92 \%$, but in regional and distant cancer it is 56 and $17 \%$, respectively [2]. Russian national guidelines in oncology and gynecology recommend managing invasive cancer with methods ranging from conization to radical surgical procedures, such as hysterectomy [3]. Vaginal radical tracheostomy is one of the less invasive procedures for nulliparous women. It is an oncologically safe fertility-preserving surgery. Human papilloma virus (HPV) is the main reason for this disease. HPV cannot be fully eliminated by surgical procedures. That is why $5.5-31.6 \%$ of cases have a relapse. Conization depth had no significant effect on the elimination HPV and precancerous lesions relapse. One of the most significant predictors of cervical lesions is damage of more than $50 \%$ of the cervical surface [4]. Tumor regression and HPV elimination can be achieved by photodynamic therapy (PDT). PDT is a noninvasive procedure to treat many oncological diseases, including early-stage invasive cervical cancer [5]. This method uses 3 main components to damage tumor cells and tissues. These components are a photosensitizer (PS), a certain wavelength of light, and oxygen [6]. A PS is intravenously injected. PS accumulation occurs in pathological and tumor tissues and is removed from healthy tissues after 2-3 h. A certain wavelength of light photoactivates stable PS and creates an excited state of PS. This PS state initiates photochemical reactions in the tumor cells. Molecular oxygen turns into singlet oxygen. Singlet oxygen is an active oxygen form. There it is inducing lipid peroxidation and programming cell death. Damage-associated molecular patterns (DAMPs) and pathogen-associated molecular patterns (PAMPs) are molecules which are produced after cell death. They are connected with toll-like receptors (TLRs) of innate immune cells. These cells synthesize cytokines and activate an innate immune response. There is a condition for the formation of active antitumor and antiviral immunity after PDT [7]. PDT makes it possible to save the cervix and preserve fertility in nulliparous women. One of these patients is described in this report.

\section{Case Report}

A 38-year-old woman presented with abdominal pain and genital tract spotting. She had no pregnancy. Her menstrual cycle was not regular and nonpainful from 15 years old. She had sex since the age of 17 years. She had had 5 sexual partners. She did not visit the gynecologist's regularly. HPV was detected in 2007 for the first time. She went with complaints to the government hospital in October 2015. There was a full examination (clinical blood test, biochemical blood test, coagulation test, urinalysis, X-ray of the chest organs, ECG, ultrasound investigation of the pelvic organs, and PAP smear). Complete blood count showed that the hemoglobin level was $127 \mathrm{~g} / \mathrm{L}$ and fibrinogen was $3.5 \mathrm{~g} / \mathrm{L}$. Erythrocytes sedimentation rate was $13 \mathrm{~mm} / \mathrm{h}$. Blood count levels were not typical for cancer patients. Adenomyosis was detected by ultrasound investigation. Hysteroscopy, dilatation, and curettage of the cervical

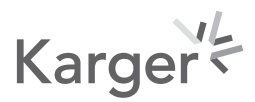




\section{Case Reports in Oncology}

\begin{tabular}{l|l}
\hline Case Rep Oncol 2021;14:506-519 \\
\hline DOI: 10.1159/000514574 & $\begin{array}{l}\odot \text { 2021 The Author(s). Published by S. Karger AG, Basel } \\
\text { www.karger.com/cro }\end{array}$ \\
\hline
\end{tabular}

Afanasiev et al.: The Multi-Course Approach of PDT to Treat Cervical Cancer
Fig. 1. Neoplasm before PDT. Exophytic neoplasm of $4 \times 6 \mathrm{~cm}$ with atypical vessels and contact bleeds.
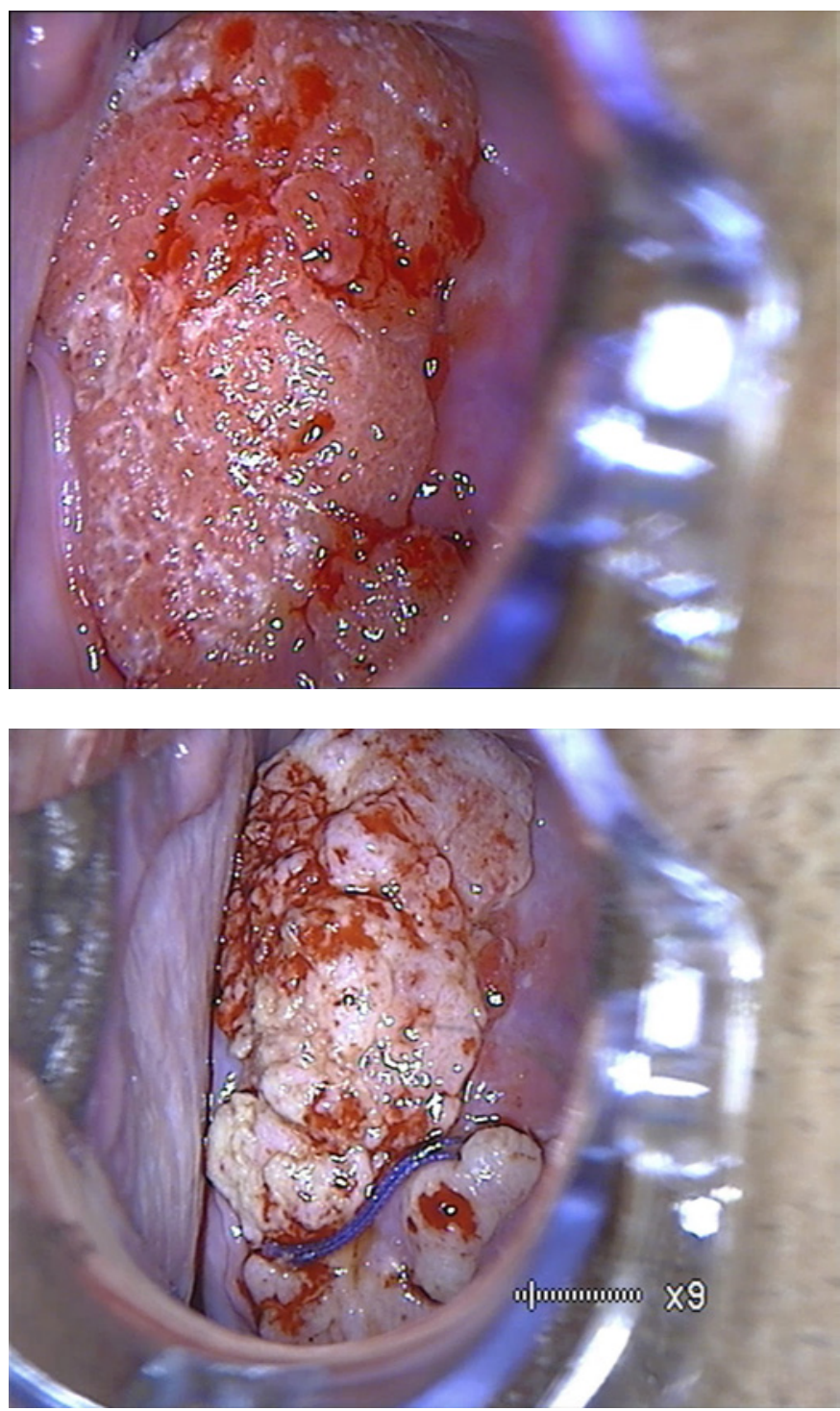

Fig. 2. Acetic test before PDT. Intensive acetowhite epithelium on the surface. Horizontal stitch on the lower pole after knife biopsy. 
Fig. 3. 8-Bit image of the cervix with acetic test. Yellow border is the cervix surface with tumor and acetic test. Red color is an area of acetowhite epithelium.

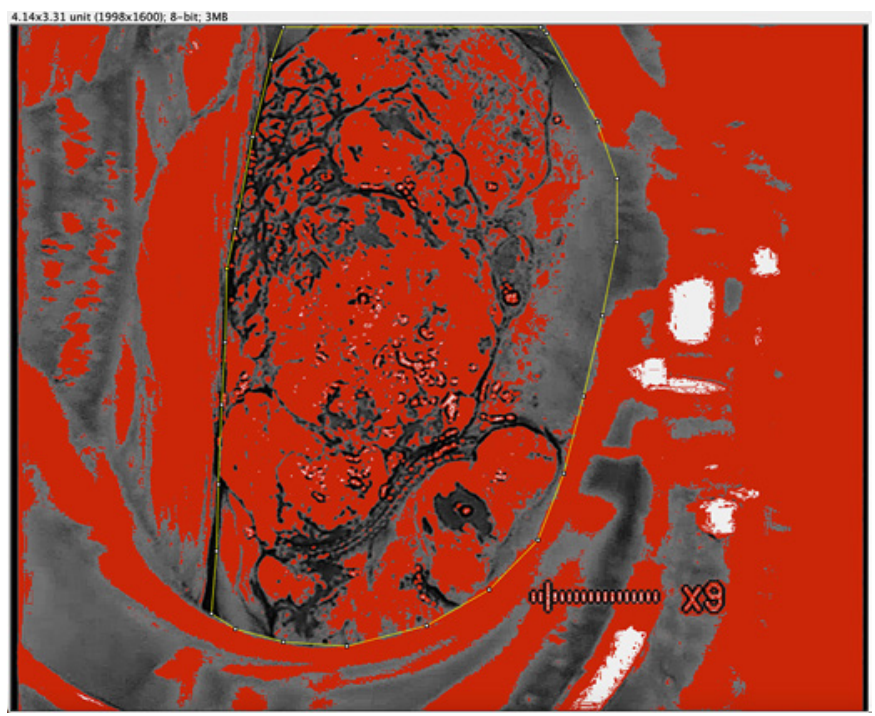

showed a highly intensive and large square of pathological tissues on the cervical surface area (shown in Fig. 3). The patient was informed about all treatment methods and chose PDT for preservation of fertility. Voluntary written informed consent was obtained for this procedure. Multicourse PDT treatment was based on the national warranty program of healthcare to cancer patients [5]. PDT was approved as a fertility-preserving treatment and we decided on 3 PDT sessions because she was not pregnant. The first session was at the end of November 2015, the second PDT session at the end of December 2015, and the third PDT session in the middle of February 2016.

\section{The First PDT Session}

General anesthesia was used for PDT. The PDT protocol included 2 stages [7]. The first stage is intravenous PS injection. Chlorin E6 (Fotoditazin by LLC VETA-GRAND, Russian Federation) was used as a PS. PS accumulation in the inner skin layers obtains a high sensitivity for sunlight. Ultraviolet (UV) protection must be provided by sunscreen. The patient used antioxidants after 3 days of each PDT session. She was observed in the low dark mode for $48 \mathrm{~h}$ after PS injection. The dark mode includes wearing sunglasses and illumination of no more than 50 lux.

\section{Irradiation Stages}

The second stage was PS photoactivation. The laser machine "Lahta-Milon" was used for cervix irradiation with $662 \mathrm{~nm}$ wavelength. The light was delivered by the cylindrical diffuser in the cervical canal (shown in Fig. 4). The irradiation part of the diffuser is $4 \mathrm{~cm}$. It irradiates the full area of the cervical canal. The cervical surface was irradiated by a macro-lens with a multi-location technique (shown in Fig. 5). The diameter of the light pole was from 2 to $3 \mathrm{~cm}$.

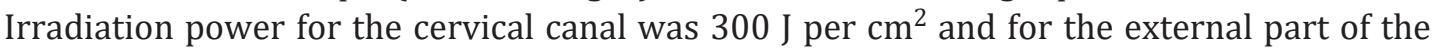
cervix it was $300 \mathrm{~J}$ per $\mathrm{cm}^{2}$ in each field with multi-location processing.

\section{Dynamic Changes in Squamous Cell Epithelium for 1 Month}

Local ischemia formed due to the photodynamic effect. A grey-white tin formed in a healthy area (shown in Fig. 6). The adverse reactions after PDT were pulling abdominal pains and a burning sensation. Nausea and vomiting were observed in $17.8 \%$ of cases. A fever was detected in the early postsurgery period. NSAIDs were used for decreasing the temperature.

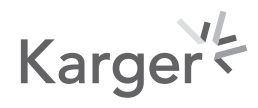




\section{Case Reports in Oncology}

Fig. 4. First stage. Cervical canal irradiation.

Fig. 5. Second stage. Cervical surface irradiation.

Fig. 6. 1 min after PDT. No coagulation necrosis indicators. The cervix surface looks blue with grey-white tin.

\begin{tabular}{l|l}
\hline Case Rep Oncol 2021;14:506-519 \\
\hline DOI: 10.1159/000514574 & $\begin{array}{l}\text { @ 2021 The Author(s). Published by S. Karger AG, Basel } \\
\text { www.karger.com/cro }\end{array}$ \\
\hline
\end{tabular}

Afanasiev et al.: The Multi-Course Approach of PDT to Treat Cervical Cancer
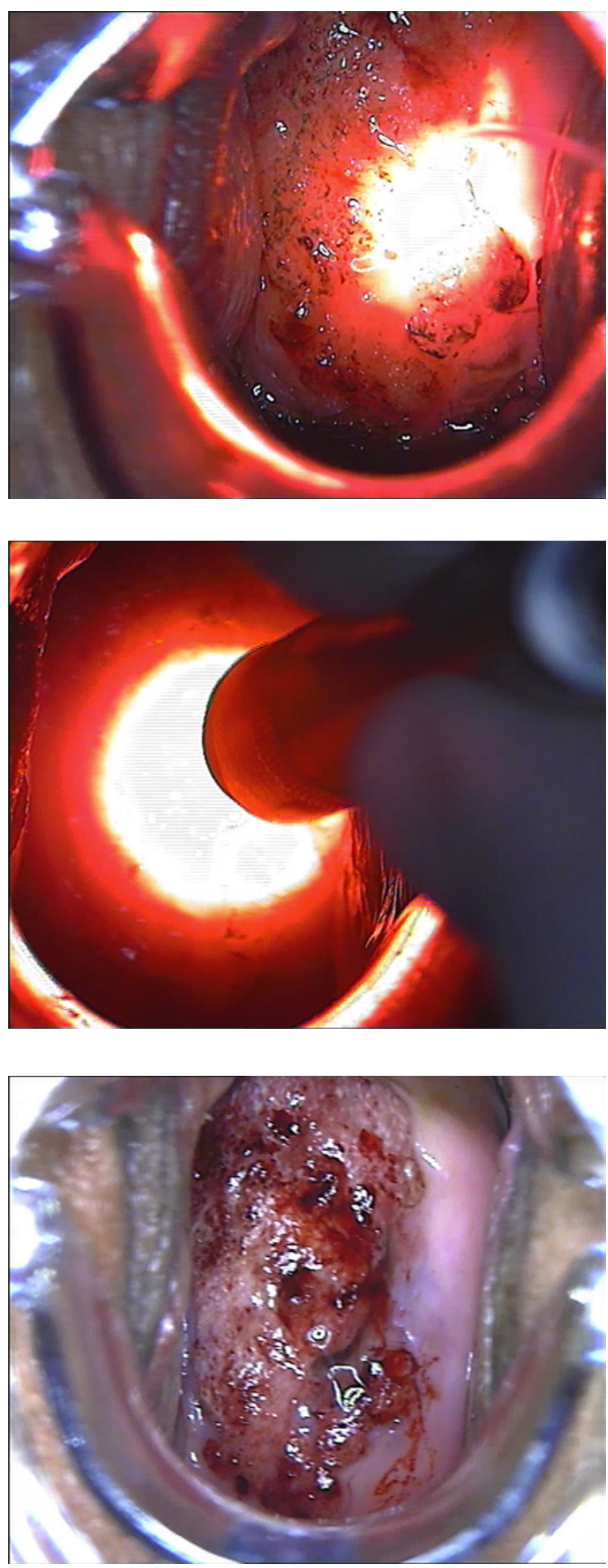

\section{Karger'}


Fig. 7. 7 days after PDT. Tumor in the resorption process. Apoptotic and necrotic masses.

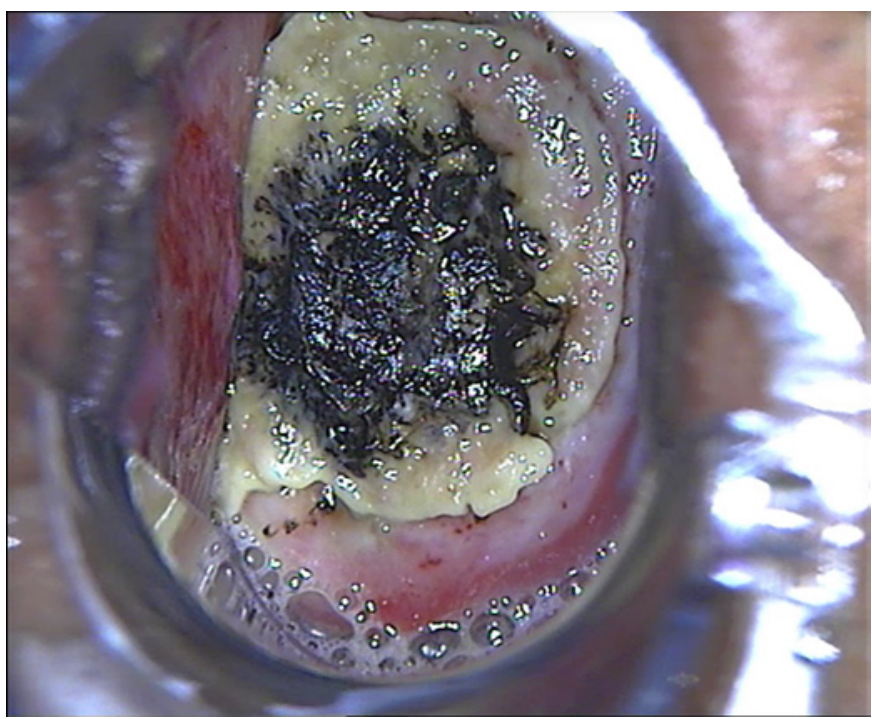

Fig. 8. Acetic test. 27 days after PDT. Erosion area around cervical cancer. Acetowhite epithelium line around erosion area.

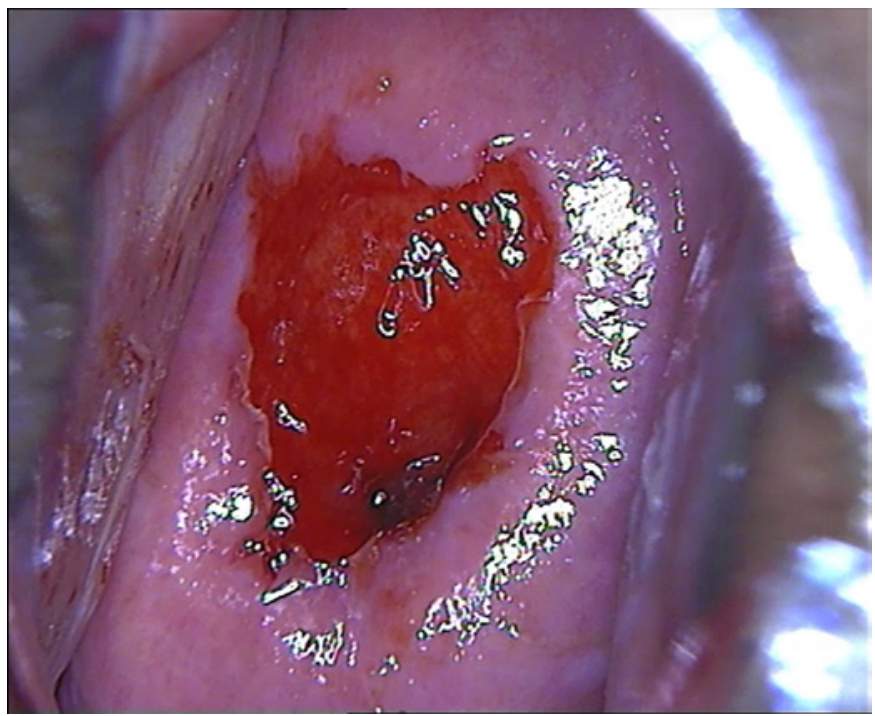

The epithelium of the organs of the female reproductive system which is HPV infected, such as the vagina and vulvar lips, has a possibility for edema and photothermal burns after PDT.

Fibrin imprinting and aseptic inflammation were forming for 1 week after PDT. These conditions are caused by apoptosis and necrosis in the tumor area (shown in Fig. 7). On the 14th to 21st day, necrotic and apoptotic masses rejection occurred. The epithelialization phase started 1 month after the PDT session (shown in Fig. 8).

Liquid-based cytology after the first PDT session had ASC-H, PAP smear result - 2nd class. Leukocyte numbers were 40-50 in a smear from the cervical canal. On MRI investigation, the cervix neoplasm was not detected, while edema of the anterior cervical part was detected. Leukocyte infiltration of the cervical canal was due to a hyperactive mucosal immunity $[9,10]$.

The same PDT protocol was used for the second PDT session. Dynamic changes in squamous cell epithelium for 1 month are shown in Figures 9-11. The cervical surface was cyanotic after 1 min of the PDT session (shown in Fig. 9). The alteration phase started 7 days after the PDT session. There were necrotic and apoptotic masses on the cervical surface 


\section{Case Reports in Oncology}

Fig. 9. 1 min after PDT. Singlet oxygen hyperoxidation. Cyanotic tissues and microthrombosis. Apoptosis was induced.

Fig. 10. 7 days after PDT. Alteration phase. Cervix surface with apoptotic and necrotic masses.

Fig. 11. 7 days after PDT. Alteration phase. Left vagina sidewall with apoptotic and necrotic masses.

\begin{tabular}{l|l}
\hline Case Rep Oncol 2021;14:506-519 \\
\hline DOI: 10.1159/000514574 & $\begin{array}{l}\text { @ 2021 The Author(s). Published by S. Karger AG, Basel } \\
\text { www.karger.com/cro }\end{array}$ \\
\hline
\end{tabular}

Afanasiev et al.: The Multi-Course Approach of PDT to Treat Cervical Cancer
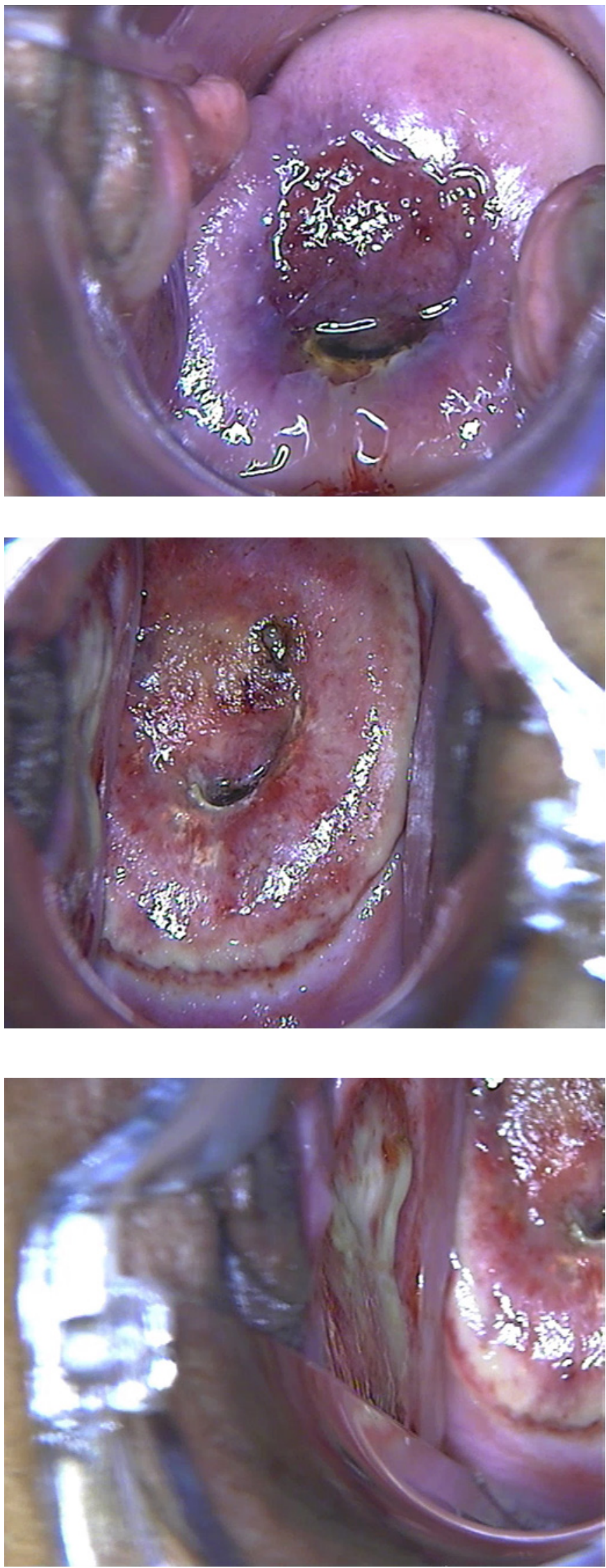

\section{Karger'}



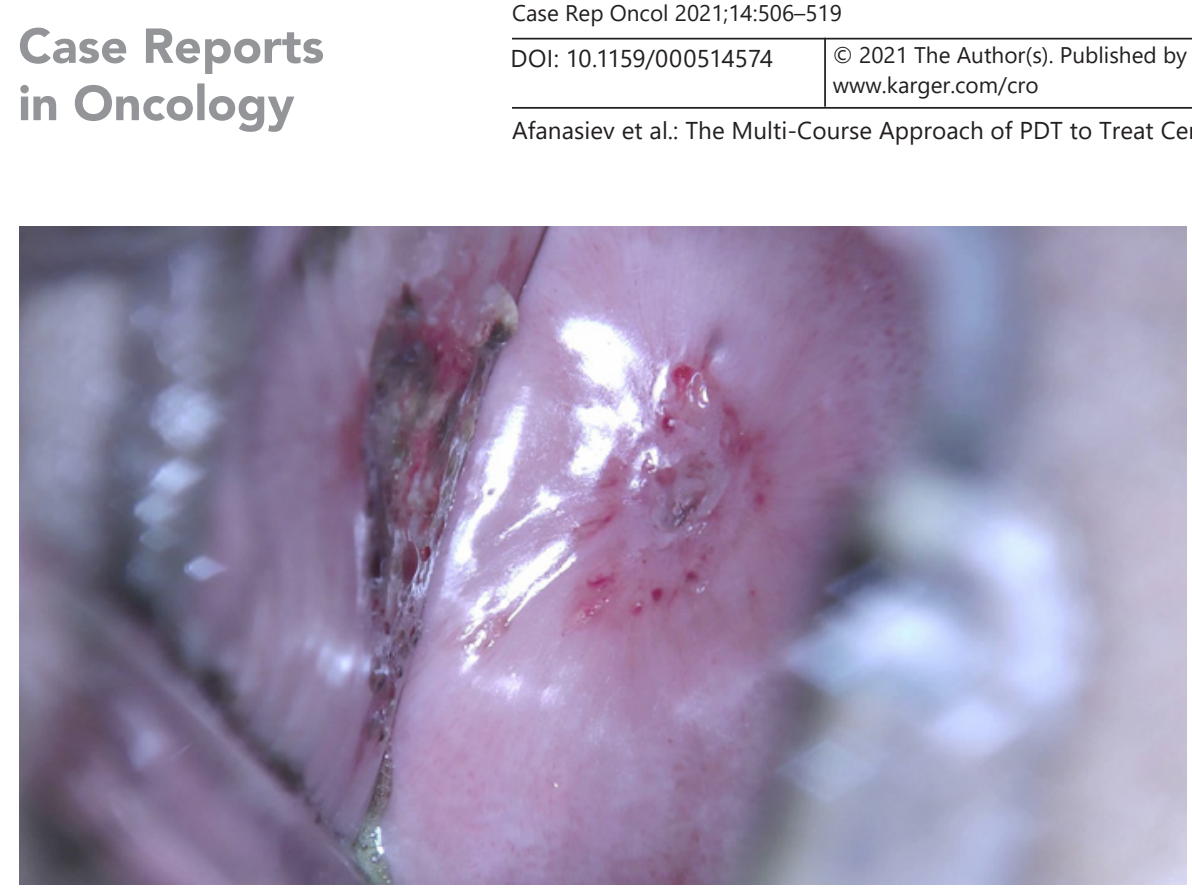

Fig. 12. Acetic test. 35 days after PDT. Endometrioid heterotopias around the cervical canal. Epithelialization process. Left vagina sidewall with PS.

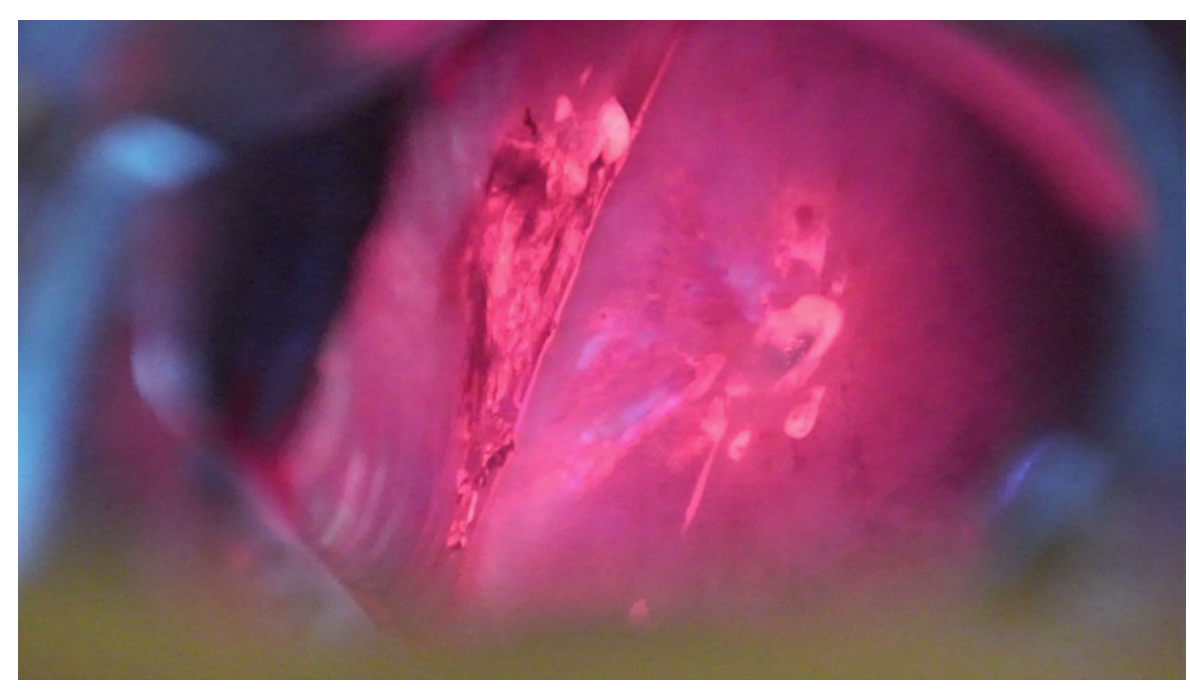

Fig. 13. Fluorescence diagnostics. 35 days after PDT. Pathological tissues fluorescence with accumulating PS.

(shown in Fig. 10) and the left vagina sidewall (shown in Fig. 11). The postsurgery period was without complications.

Liquid-based cytology after the second PDT session was negative for intraepithelial lesion or malignant (NILM). The left vagina sidewall had an inflammation process (shown in Fig. 12). Fluorescence diagnostics were used to control the efficacy of previous procedures. PS accumulation was controlled by fluorescent diagnostics. UV-light was generated by the apparatus "AFS" with $400 \mathrm{~nm}$ wavelength of light (LLC Polaronic, Russian Federation). A yellow light filter was installed in the colposcope for excluding UV-light and fixate red light fluorescence. Red fluorescence was present after PS accumulation (shown in Fig. 13). Pathological fluorescence showed inflammation of tissues. We decided to hold a third PDT session.

The same PDT protocol was used for the third PDT session. Dynamic changes in squamous cell epithelium for 7 days are shown in Figures 14-16. Reaction of the tissues of the cervix 


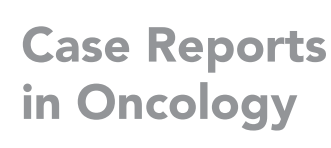

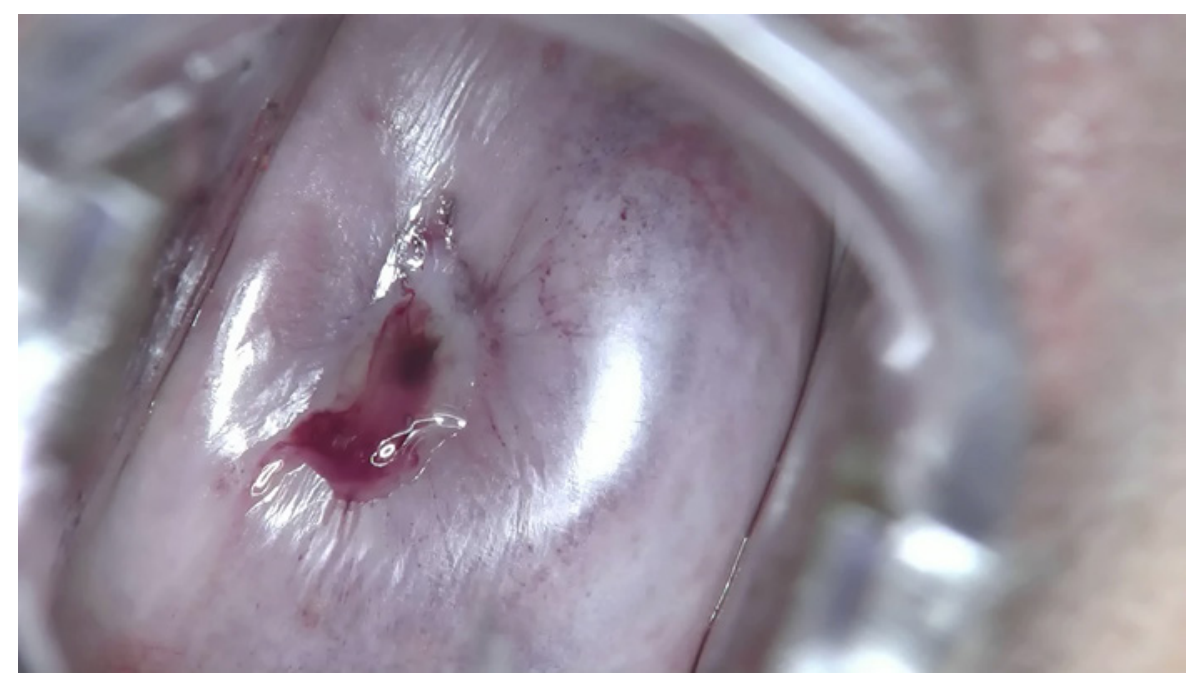

Fig. 14. 1 min after PDT. Cervix surface. Cyanosis area. Microthrombosis.

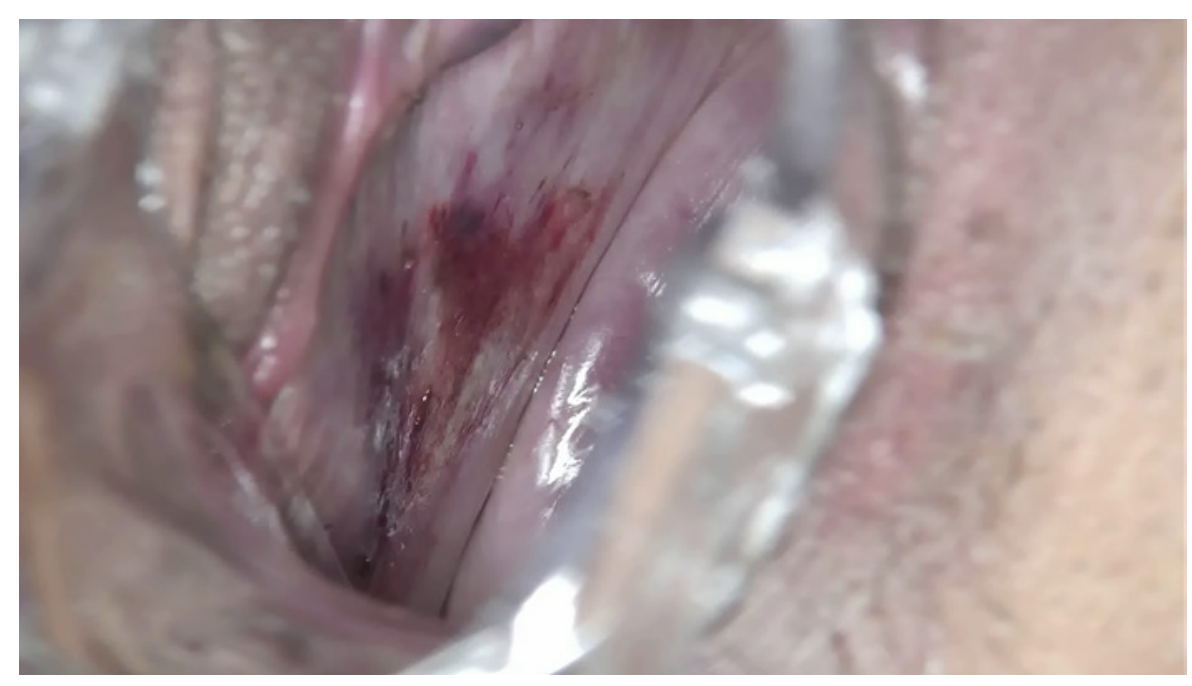

Fig. 15. 1 min after PDT. Left vagina sidewall. Cyanosis area. Microthrombosis.

and left vagina sidewall after PDT is shown in Figures 14 and 15. Microthrombosis-related ischemia is a reason for the cyanosis area on the cervical surface after PDT. Alteration phase with rejection of necrotic and apoptotic masses after 7 days of PDT is shown in Figure 16.

The patient had a checkup after treatment 4 times (3rd, 12th, 24th, and 60th months). Cervix histological investigation results detected chronic HPV-related cervicitis without atypical squamous cells and chronic mild colpitis 3 months after the third PDT session. Liquidbased cytology after the third PDT session was NILM. On MRI visualization of the pelvic organs no relapse was detected and the neoplasm regressed. Squamous cell carcinoma (SCC) antigen was equal to $0.3 \mathrm{ng} / \mathrm{mL}$.

The condition of the cervix 12 months after PDT is shown in Figure 17. Digene test 12 months after the third PDT session calculated a weak viral load ( 0.45 relative units). Colposcopy had no acetowhite epithelium and iodine negative areas (shown in Fig. 18, 19, 


\section{Case Reports in Oncology}

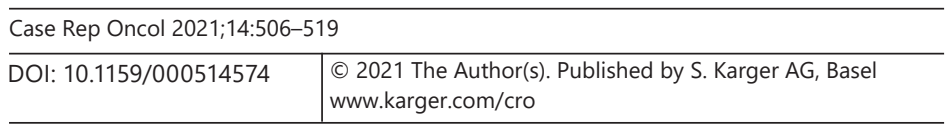

Afanasiev et al.: The Multi-Course Approach of PDT to Treat Cervical Cancer

Fig. 16. 7 days after PDT. Alteration phase. Necrotic and apoptotic masses.
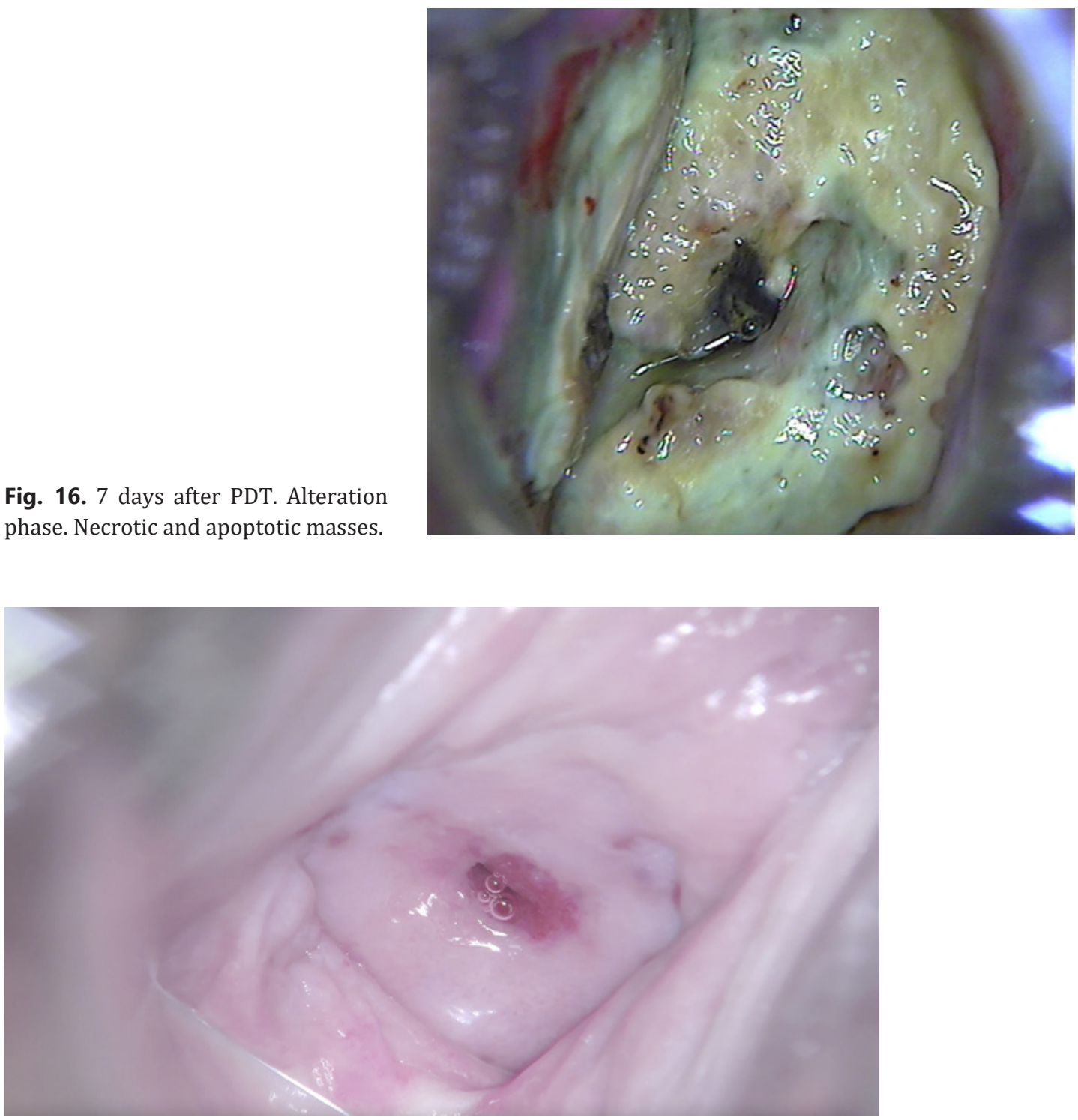

Fig. 17. Full epithelialization. Metaplastic squamous cell epithelium around the cervical canal. TZ type 2. Endometrioid heterotopia at 1 and 11 hours.

respectively). Liquid-based cytology result 12 months after the third PDT session was NILM. PAP smear had as a result 1 class. Leukocyte numbers were 10-12 in a smear from the cervical canal. Mucosal immunity activity was on normal status $[9,10]$. The patient has been doing a checkup every year since 2016.

Her pregnancy occurred 3 years and 8 months after the first PDT session. The pregnancy was without complications. She had operative delivery by Cesarean section in April 2020. Operative delivery was chosen to prevent cervical rupture. The patient went to investigational control 8 months after labor. Colposcopy had no acetowhite epithelium and iodinenegative areas (shown in Fig. 20-22).

The liquid-based cytology 60 months after PDT sessions detected NILM (a weak columnar epithelium proliferation). PAP smear had as a result 1 class. Leukocyte numbers 

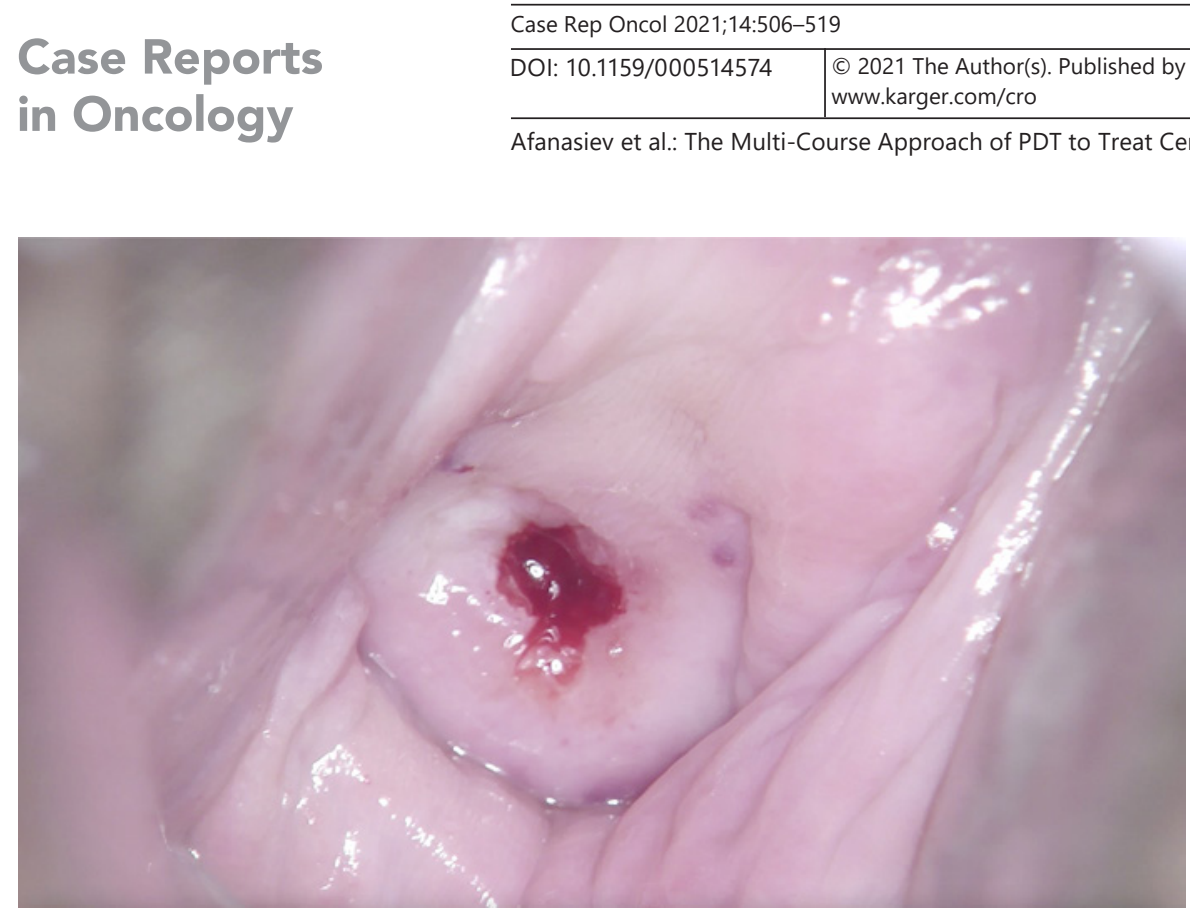

Fig. 18. Acetic test. No acetowhite epithelium on the cervix surface. Bleeding after cytobrush effect. TZ type 2 .

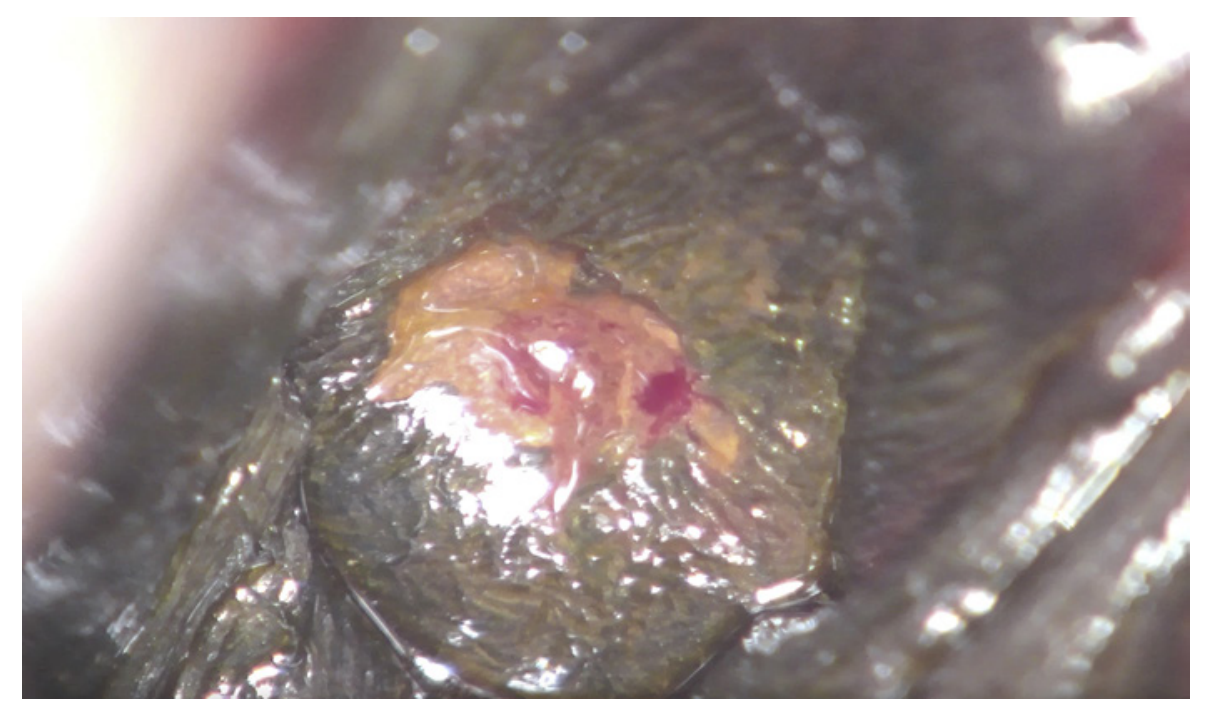

Fig. 19. Schiller's test. Iodine-negative areas are not detected. Squamous cell epithelium after epithelialization phase around the cervical canal. TZ type 2.

were $0-3$ in a smear from the cervical canal, a normal activity of mucosal immunity $[9,10]$. Digene test calculated a weak viral load ( 0.22 relative units) 5 years after PDT. MRI visualization of the pelvic organs did not detect any pathology in the cervix. There was a ridge in the lower part of the uterus after the Cesarean section. IndGV was equal to 0.22 units. It showed lowly intensive and big square pathological tissues on the cervical surface area (shown in Fig. 23). 


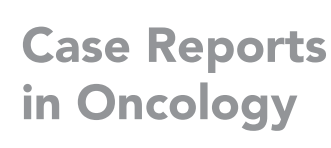

Case Rep Oncol 2021;14:506-519

DOI: $10.1159 / 000514574$

(c) 2021 The Author(s). Published by S. Karger AG, Basel www.karger.com/cro

Afanasiev et al.: The Multi-Course Approach of PDT to Treat Cervical Cancer

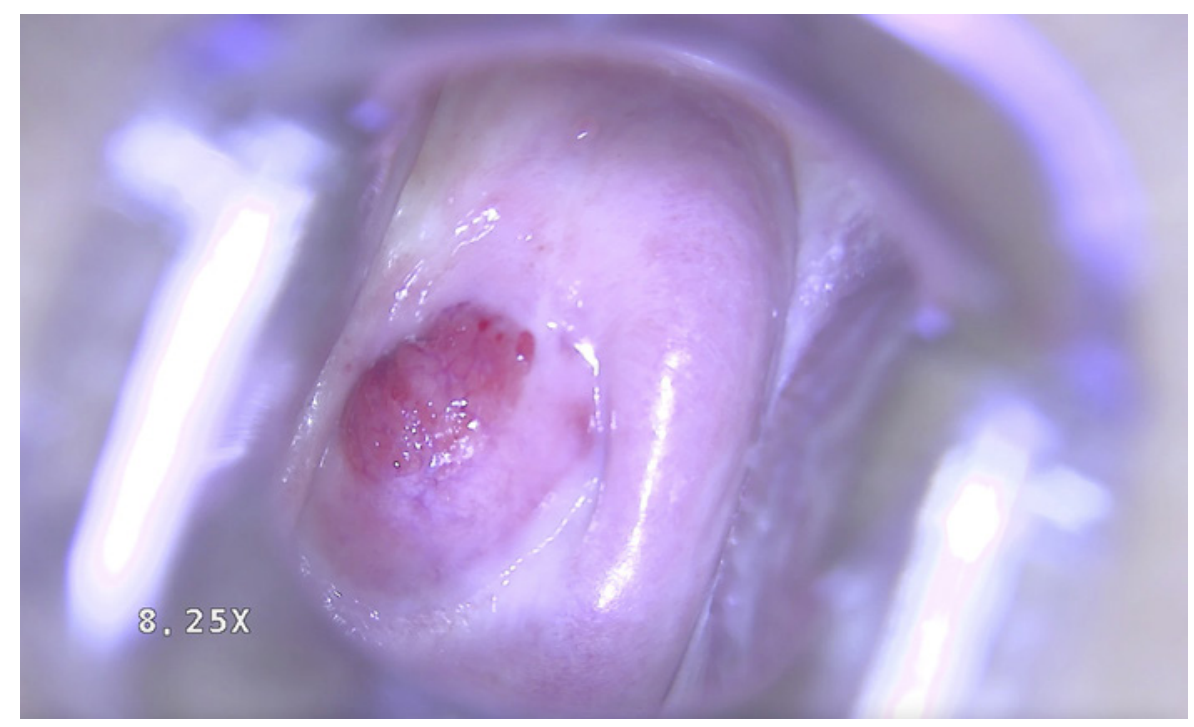

Fig. 20. Ectopia of the uteri cervix.

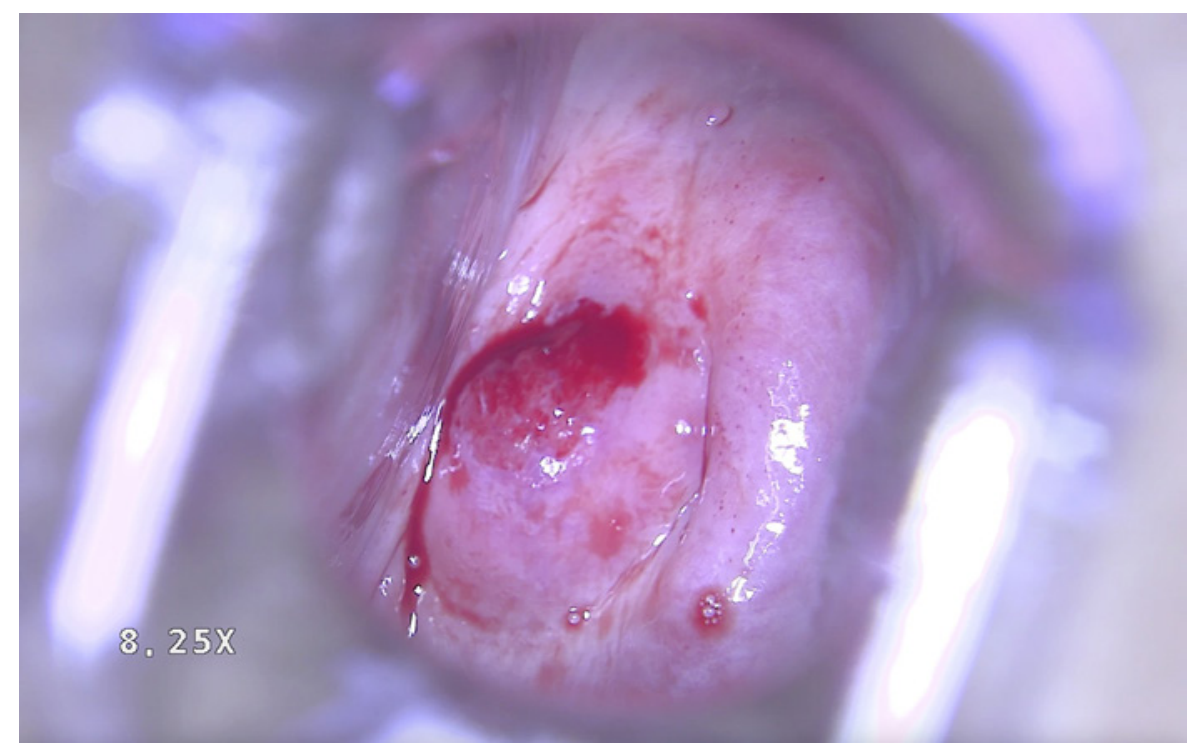

Fig. 21. No acetowhite epithelium. Bleeding after cytobrush effect.

\section{Conclusion}

PDT is an organ-saving treatment method for preserving fertility. This is a noninvasive treatment for invasive cervical cancer. HPV is the main reason for cervical cancer. PDT activates mucosal, antitumor, and antiviral immunity. Photodynamic reactions initiate immune cells by cytokines and TLRs $[7,9,10]$. PDT normalized the mucosal immunity in the cervix. A full preoperative examination provides clinical details for understanding the level of the nonsurgical intervention and numbers of PDT sessions. Our 5-year-term experience showed that patients with squamous cell lesions and early-stage invasive cervical cancer needed to 

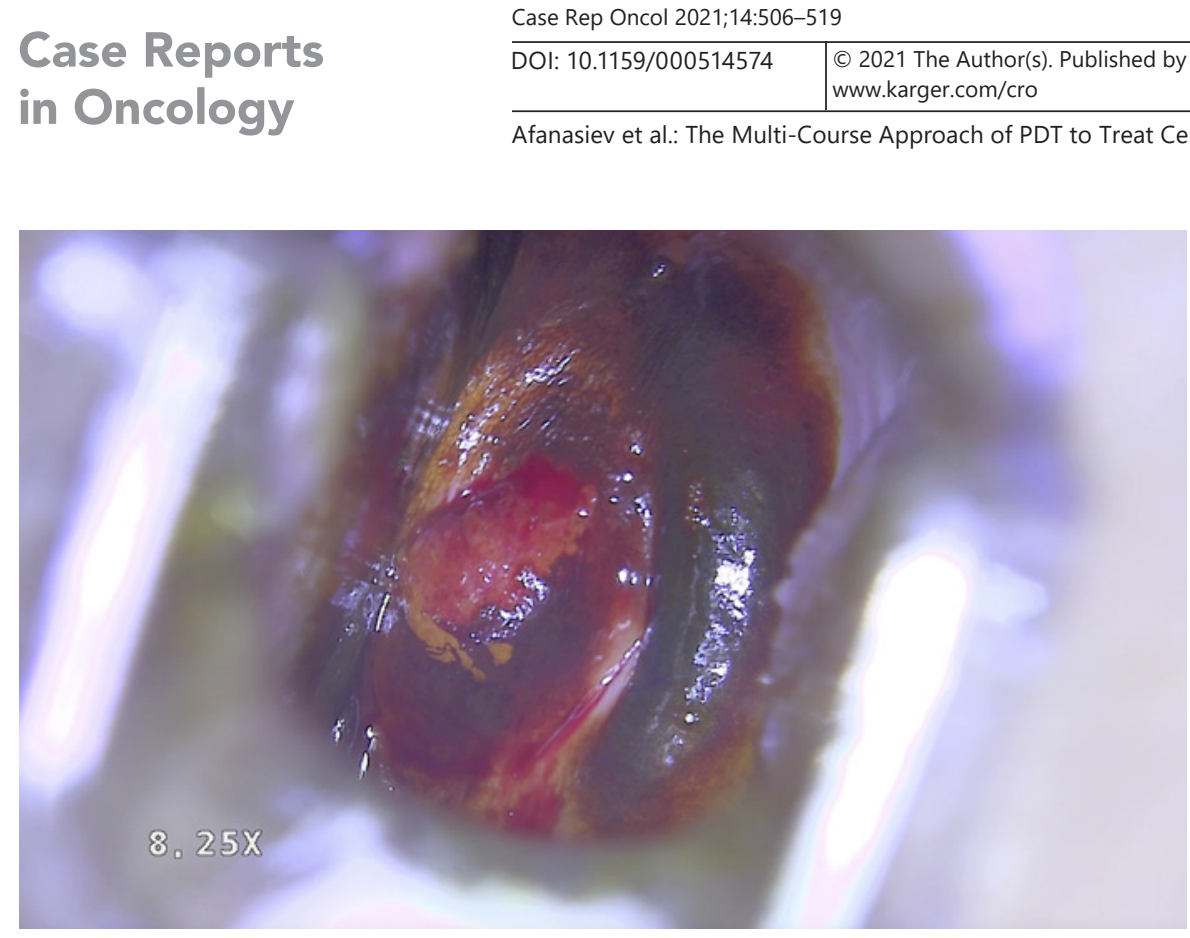

Fig. 22. Iodine-positive area.

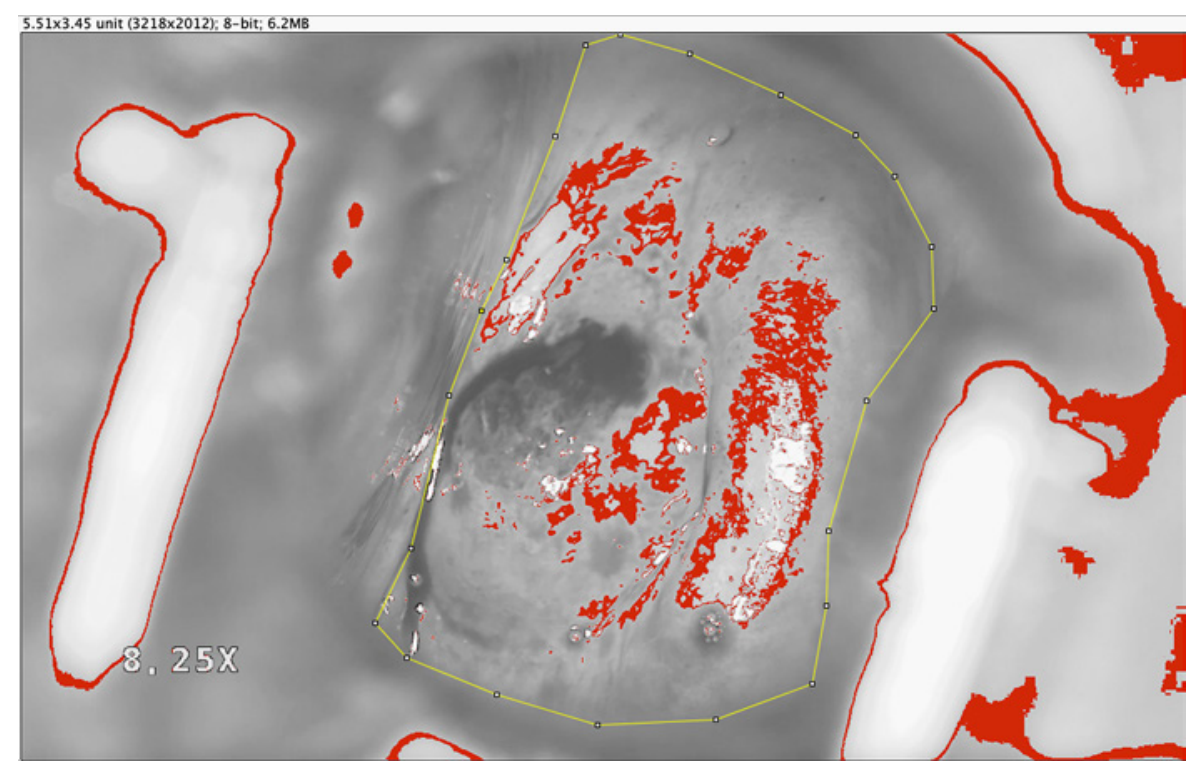

Fig. 23. 8-Bit image of the cervix with acetic test. Yellow border is the cervix surface with tumor and acetic test. Red color is an area of white light reflexion.

have 1 PDT session and multi-course PDT, respectively. This patient has a 5-year remission without episodes of relapse. PDT allowed to avoid vaginal radical trachelectomy and to preserve fertility. The patient supports the 8-month-old baby. PDT can be a method of choice for patients who have never been pregnant. 


\section{Case Reports in Oncology}

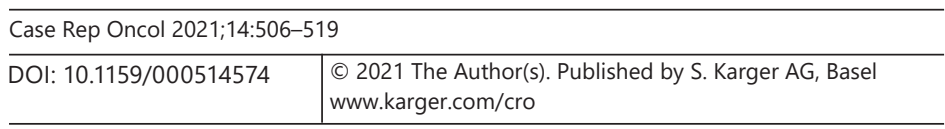

Afanasiev et al.: The Multi-Course Approach of PDT to Treat Cervical Cancer

\section{Statement of Ethics}

The authors have no ethical conflicts to disclose. Ethics approval was not required for this report (ethical committee of the I.M. Sechenov Moscow State Medical University). Written informed consent was obtained from the legal guardians of the patient (parent) for publication of this case report and any accompanying images.

\section{Conflict of Interest Statement}

The authors have no conflicts of interest to declare.

\section{Funding Sources}

There were no funding resources.

\section{Author Contributions}

Dr. Maxim Afanasiev treated the patient, made the colposcopy investigation recording, and reviewed the clinical part of the manuscript. Dr. Alexander Dushkin wrote the manuscript and made the digital analysis. Tatyana Grishacheva made the calculation for the treatment protocol and reviewed the manuscript. Dr. Stanislav Afanasiev reviewed the introduction and conclusion of the manuscript. Dr. Yuri Nesvizhsky reviewed the introduction and conclusion of the manuscript. Dr. Alexander Karaulov approved the final version to be published. Dr. Andrey Pylev helped to cooperate with all services in the "European Clinic".

\section{References}

1 International Agency for Research on Cancer [Internet]. GLOBOCAN 2018. Available from: https://www.iarc. fr/

2 American Cancer Society [Internet]. Atlanta: Survival Rates for Cervical Cancer. [cited 2020 Jan 3]. Available from: https://www.cancer.org/cancer/cervical-cancer/detection-diagnosis-staging/survival.html

3 Russian Clinical Guidelines for Cervical Cancer (C53) 2020 [Internet]. Available from: https://oncology-association.ru/files/clinical-guidelines-2020/rak_shejki_matki.pdf

4 Chang H, Hong JH, Lee JK, Cho HW, Ouh YT, Min KJ, et al. Programmed death-1 (PD-1) expression in cervical intraepithelial neoplasia and its relationship with recurrence after conization. J Gynecol Oncol. 2018;29(3): e27-14.

5 Decree of the Government of Russia of December 19th, 2015 No. 1382 "On the program of state guarantees for free provision of medical care to citizens for 2016 and for the planning period 2017 and 2018."

6 Castano AP, Demidova TN, Hamblin MR. Mechanisms in photodynamic therapy: Part one - Photosensitizers, photochemistry and cellular localization. Photodiagnosis Photodyn Ther. 2004;1(4):279-93.

7 Karaulov AV, Afanasiev MS, Petrischev NN, et al. Photodynamic therapy: immunomodulating method in oncogynecology. In: Afanasyev MS, Karaulov AV, Alyoshkin M, editors. Textbook for the system of postgraduate professional education of doctors. Moscow: LLC Contact; 2020. pp. 140.

8 Application for an invention No. 2020120982 "Method for determining the degree of the cervical dysplasia" (priority 25.06.2020 RU).

9 Karaulov AV, et al. Mucosal immunity and colonization resistance of open mucous membranes a person in norm and in pathological conditions. In: Karaulov AV, editor. Textbook for postgraduate professional education of doctors. Moscow, Publishing House of the I.M. Sechenov First Moscow State Medical University; 2017. pp. 76.

10 Afanasiev MS, Karaulov AV, Aleshkin AV, et al. The role of innate immunity in pathogenesis, molecular genetic mechanisms, diagnosis and treatment of urogenital infection in women. Moscow: M. Publishing House Dynasty; 2019. pp. 274. ISBN 978-5-98125-111-5. 